\title{
Severe Phymatous Rosacea of the Nose, Cheeks, and Chin Treated With Hydrosurgery
}

\author{
Julie M. Bittar, MD; Stephen J. Kovach, MD; Chérie M. Ditre, MD
}

\section{PRACTICE POINTS}

- Phymatous rosacea is a rare disfiguring disease that most commonly affects men and can have considerable effects on a patient's physical, mental, and social well-being.

- Treatment of phymatous rosacea usually is surgical; however, no consensus guidelines exist for best surgical management.

- The Versajet II Hydrosurgery System can be useful and effective for the treatment of phymatous rosacea, not only on the nose but elsewhere on the face.
Phymatous rosacea is a rare and severe form of rosacea that manifests as disfiguring soft-tissue hypertrophy and sebaceous gland hyperplasia and fibrosis. Most cases are surgically treated; surgical modalities vary, however, ranging from cryosurgery to conventional excision, and consensus guidelines for surgical management do not exist. The Versajet II Hydrosurgery System (Smith-Nephew) is a highpressure, pulsatile lavage system. We present the case of a 75-yearold man with severe phymatous rosacea of the nose, cheeks, and chin who was successfully treated with the Versajet II Hydrosurgery System, yielding excellent contouring.

Cutis. 2020;106:37-39.

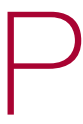

hymatous rosacea is a rare and severe form of rosacea that manifests as disfiguring soft-tissue hypertrophy and hyperplasia as well as fibrosis of the sebaceous glands. ${ }^{1}$ Treatments for phymatous rosacea include pharmacotherapeutic and surgical modalities; most cases are treated surgically. Surgical modalities vary, ranging from cryosurgery to conventional excision, and consensus guidelines for surgical management do not exist because data are largely limited to case reports and small case series. ${ }^{2}$ The Versajet II Hydrosurgery System (Smith-Nephew) is a high-pressure, pulsatile lavage system that has been used for phymatous rosacea and then only for rosacea of the nose (rhinophyma). We present the case of a patient with phymatous rosacea of the nose, cheeks, and chin who was successfully treated with the Versajet II Hydrosurgery System beyond just the nose region.

\section{Case Report}

A 75-year-old man presented to the dermatology clinic for evaluation of severe phymatous rosacea of the nose, cheeks, and chin that had been present for several years. Examination revealed verruciform, thickened, erythematous skin of the nose, cheeks, and chin; marked blue-gray hyperpigmentation on the neck and hands; generalized facial redness; and cystic and depressed scars (Figure 1). The patient had been treated with topical metronidazole without response, and isotretinoin worsened the symptoms. He also was taking minocycline but stopped it at our request because of concern that the drug was causing the blue-gray hyperpigmentation. The patient was referred to plastic surgery and tangential excision was recommended. Fractional ablative laser therapy was considered but deferred because the patient wanted quicker results.

Dr. Bittar is from the Indiana University School of Medicine, Indianapolis. Dr. Kovach is from the Division of Plastic Surgery, and Dr. Ditre is from the Department of Dermatology, both from Penn Medicine at Radnor, Pennsylvania.

The authors report no conflict of interest.

Correspondence: Chérie M. Ditre, MD, Penn Medicine at Radnor, 250 King of Prussia Rd, Radnor, PA 19087 (Cherie.Ditre@uphs.upenn.edu). 

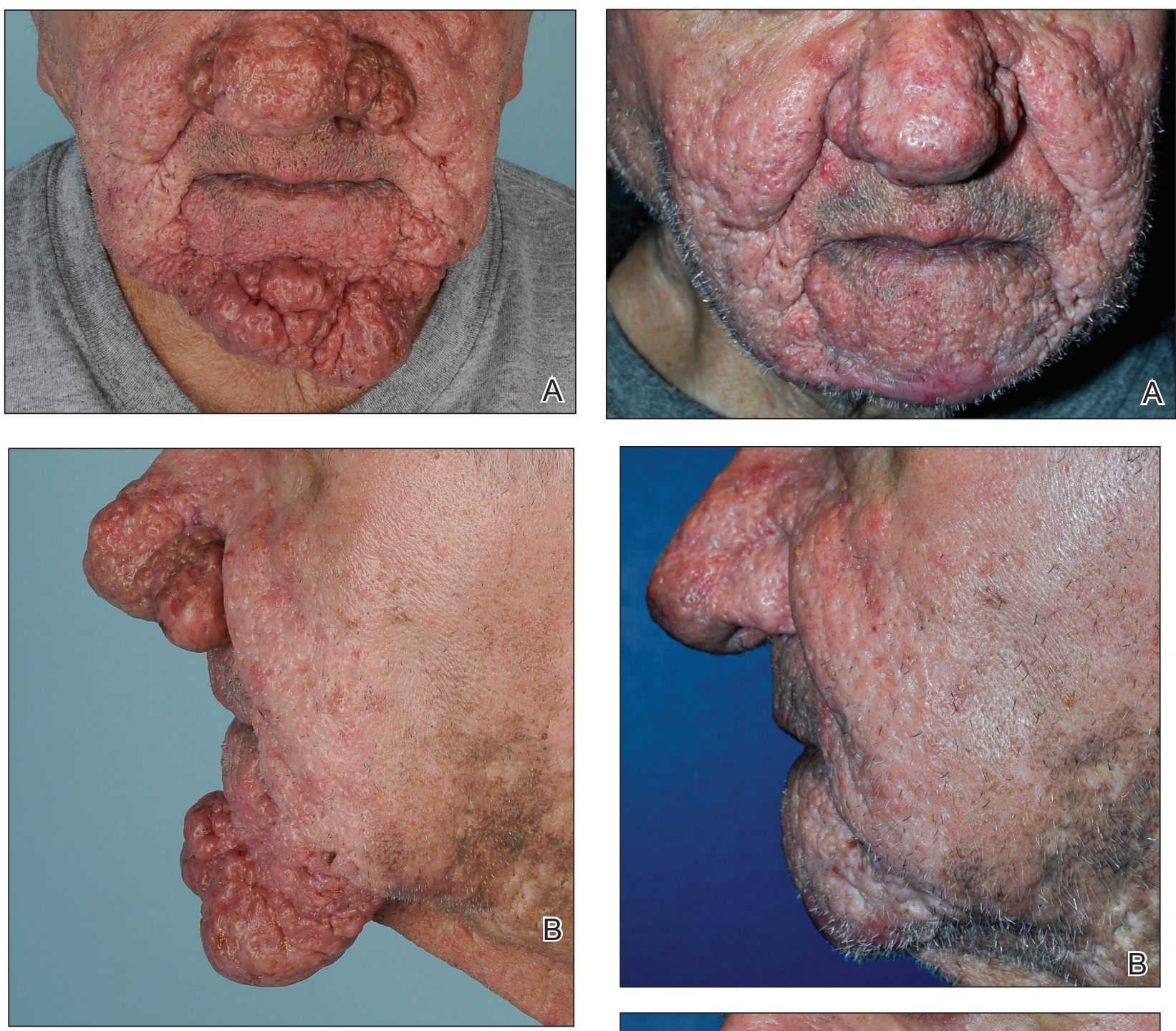

FIGURE 1. A and B, Severe phymatous rosacea of the nose, cheeks, and chin, as well as generalized rosacea and hyperpigmentation of the sclera.
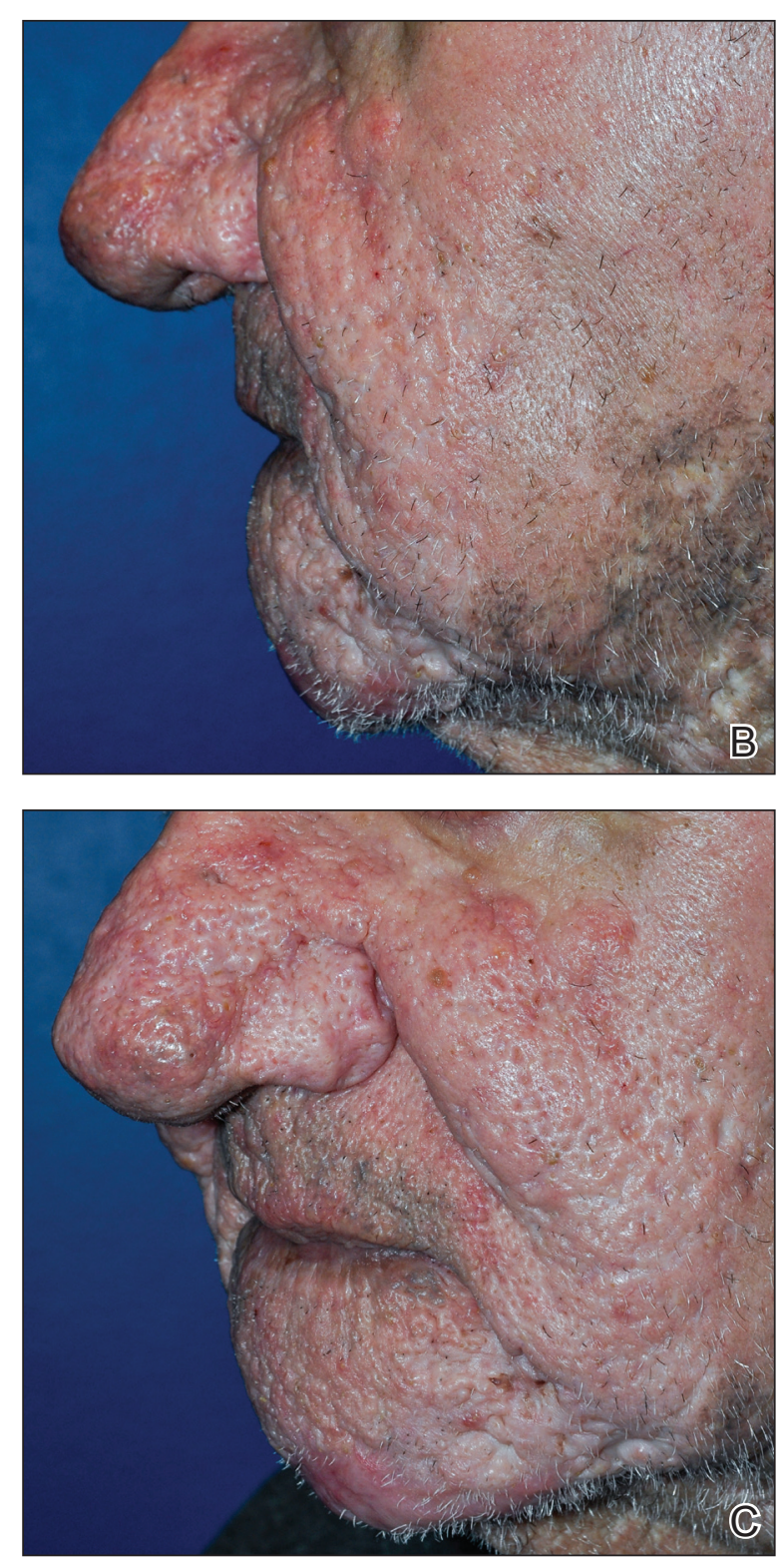

The patient received tangential excision of the phymatous areas of the chin, bilateral cheeks, and nose with the Versajet II Hydrosurgery System until a pleasing contour was noted. At 1-month follow-up, the patient had an excellent contour of the nose, cheeks, and chin (Figure 2).

\section{Comment}

Phymatous rosacea is a rare disfiguring disease that most commonly presents on the nose but also can affect the chin, cheeks, eyelids, ears, and forehead. Incidence is greater in individuals of Scottish descent and in men due to the influence of androgens. The etiology of the condition is unknown. ${ }^{1}$

Aside from clinical findings of hyperplastic and fibrotic sebaceous glands in conjunction with enlargement of the affected facial areas, histopathologic

FIGURE 2. A-C, Marked improvement in facial contour after tangential excision of phymatous skin of the nose, cheeks, and chin. 
findings of phymatous rosacea vary but typically include hypertrophy of subcutaneous tissue, enlarged sebaceous ducts filled with keratin and sebum, atrophy of the dermis, and abnormal vascular development in the form of telangiectases.

Phymatous rosacea adversely affects patients' physical, mental, and social well-being. Left untreated, it can cause nasal obstruction and recurrent bacterial infections. Furthermore, because of the potential extent of facial deformity, phymatous rosacea can be highly stigmatizing. ${ }^{3}$ Nonmelanoma skin cancers have been reported within phymatous skin, but evidence of an association between the 2 diseases remains inconclusive. ${ }^{4}$ Excised tissue from our patient was not submitted to pathology for analysis.

Given the far-reaching physical and psychological consequences of phymatous rosacea, treatment is critical but, regrettably, challenging. Although medical and surgical interventions exist, surgery is the most common practice. Oral isotretinoin may help, but many cases are recalcitrant, as was the disease in our patient. Therefore, procedural remedies often are sought, including scalpel excision, cryosurgery, argon laser, $\mathrm{CO}_{2}$ laser, dermabrasion, and electrocautery. ${ }^{2}$

Our patient underwent Versajet II Hydrosurgery System treatment of the phymatous rosacea on the nose, cheeks, and chin. Versajet is not yet commonly used to treat phymatous rosacea, likely due to the upfront cost of obtaining a new device, lack of physician familiarity, and few reports of its use for phymatous skin. A search of PubMed, EMBASE, and the Web of Science using the terms Rosacea AND (Versajet OR Hydrosurgery) yielded only 6 cases of rosacea treated by hydrosurgery; all were limited to rhinophyma and reported excellent cosmetic and functional results. ${ }^{5-10}$ Our case was unique in that hydrosurgery was used to treat phymatous rosacea beyond the nose.

Hydrosurgery has many advantages in the treatment of phymatous rosacea and other conditions in which surgical debridement is necessary, such as burns and wounds. A randomized clinical trial demonstrated that hydrosurgery is more cost-effective than conventional excision because of decreased operative time and intraoperative blood loss, fewer debridement procedures, and fewer postoperative complications. ${ }^{11}$

Rennekampff et $\mathrm{al}^{12}$ showed that Versajet debridement is superior to conventional surgery in contouring facial and acral sites and has a lower probability of infection. They proposed that by running a highly pressurized constant stream of saline across the device, Versajet clears blood and debris from the surgical site during excision. ${ }^{12}$
Hydrosurgical debridement also has been shown to reduce Staphylococcus aureus inoculate levels from in vitrocontaminated equine models significantly more than conventional debridement methods $(P<.05) .{ }^{13}$

Versajet surgery appears to be well tolerated, with side effects comparable to those of classic surgical excision. A randomized controlled trial in burn patients in which treatment with Versajet was compared to traditional debridement found no significant difference in postoperative pain, healing time, and contracture rate..$^{13}$

Overall, tangential excision of our patient's phymatous rosacea using the Versajet II Hydrosurgery System yielded excellent contouring. However, due to the paucity of literature on the subject, it is difficult to discern the optimal treatment modality. Therefore, more research-ideally randomized trials - should be pursued to examine the comparative effectiveness of different interventions for phymatous rosacea.

\section{REFERENCES}

1. Curnier A, Choudhary S. Rhinophyma: dispelling the myths. Plast Reconstr Surg. 2004;114:351-354.

2. Sadick H, Goepel B, Bersch C, et al. Rhinophyma: diagnosis and treatment options for a disfiguring tumor of the nose. Ann Plast Surg. 2008;61:114-120.

3. Dirschka T, Micali G, Papadopoulos L, et al. Perceptions on the psychological impact of facial erythema associated with rosacea: results of international survey. Dermatol Ther (Heidelb). 2015;5:117-127.

4. Lazzeri D, Colizzi L, Licata G, et al. Malignancies within rhinophyma: report of three new cases and review of the literature. Aesthetic Plast Surg. 2012;36:396-405.

5. Dunne JA, Saleh DB, Rawlins JM. Management of rhinophyma with Versajet $^{\mathrm{TM}}$ and ReCell®. Br J Oral Maxillofac Surg. 2013;51:e282-e284.

6. Yildiz K, Kayan BR, Dulgeroglu T, et al. Treatment of rhinophyma with the Versajet ${ }^{\mathrm{TM}}$ Hydrosurgery System and autologous cell suspension (ReCELL®): a case report. J Cosmet Laser Ther. 2018;20:114-116.

7. Nicolas J, Garmi R, Labbé D, et al. The role of Versajet in the surgical treatment of rhinophyma. case report. Ann Chir Plast Esthet. 2009;54:78-81.

8. Novati FC, Franchi A, Roggio T, et al. Treatment of a double-giant rhinophyma with electrocautery and Versajet Hydrosurgery System. Ann Ital Chir. 2015;86. pii: S2239253X15023269.

9. Taghizadeh R, Mackay SP, Gilbert PM. Treatment of rhinophyma with the Versajet Hydrosurgery System. J Plast Reconstr Aesthet Surg. 2008;61:330-333.

10. Wong WL, Wong She R, Mathy JA. Rhinophyma treatment using Versajet Hydrosurgery. ANZ J Surg. 2017;87:E331-E332.

11. Liu J, Ko JH, Secretov E, et al. Comparing the hydrosurgery system to conventional debridement techniques for the treatment of delayed healing wounds: a prospective, randomised clinical trial to investigate clinical efficacy and cost-effectiveness. Int Wound J. 2015;12:456-461.

12. Rennekampff H-O, Schaller H-E, Wisser D, et al. Debridement of burn wounds with a water jet surgical tool. Burns. 2006;32:64-69.

13. Skarlina EM, Wilmink JM, Fall N, et al. Effectiveness of conventional and hydrosurgical debridement methods in reducing Staphylococcus aureus inoculation of equine muscle in vitro. Equine Vet J. 2015; 47:218-222. 